\title{
Wie heilig waren die Heiligen Spiele? Eine Suche NACH IHREN KULTISCHEN FUNKTIONEN \\ How Sacred were the Sacred Games? Seeking out their Cultic Functions
}

Werner Petermandl

KarL-FranZENS-UNIVERSITÄT GRAZ WERNER.PETERMANDL@UNI-GRAZ.AT

\section{ÜBERSICHT}

Im modernen Fachschriftum werden die bedeutenden Agone der griechischen Antike häufig als Heilige Spiele bezeichnet. Aber was berechtigt zu solch einer Charakterisierung? Ist es lediglich die Tatsache, dass an sich profane Wettkämpfe im Rahmen von religiösen Festen stattfanden, oder steht vielmehr dahinter, dass die Wettbewerbe selbst kultischen Charakter hatten, ja sakrale Handlungen waren? In diesem Aufsatz wird die Frage gestellt, ob die antiken Quellen tatsächliche kultische Funktionen der Wettkämpfe bezeugen können - und negativ beantwortet.

\section{Abstract}

In modern scholarly work, the most important agones of Greek antiquity are commonly referred to as Sacred or Holy Games. But how can such a characterization be justified: only by the mere fact that those contests, albeit secular, took place within the programme of religious festivals or rather by the cultic character of the contests themselves, actually being sacred ceremonies? The article seeks to address the question whether the ancient sources can attest actual cultic functions of the contests and comes to a negative result.
KEY WORDS

Antikes Griechenland, Sport, Agon, Wettkämpfe, Kult, Religion
KEY WORDS

Ancient Greece, sport, agon, contests, cult, religion 
Vor Nicht AllzU langer Zeit hatte ICH eine Auseinandersetzung mit einem Kollegen, dessen Name hier ungenannt bleiben soll, der behauptet hatte, dass antike athletische Agone niemals mit modernen Sportwettkämpfen verglichen werden könnten. Denn die antiken Veranstaltungen wären von ganz anderer Natur als die heutigen - sie wären religiös. Ich habe ihm hierin nicht zustimmen können. Als ich nun die Einladung zu dieser Tagung erhielt, ${ }^{1}$ erinnerte ich mich an diese Diskussion sowie an zahlreiche entsprechende Aussagen im Fachschrifttum, und entschloss mich, die Fragestellung bei dieser Gelegenheit erneut einer Betrachtung zu unterziehen.

Beginnen wir mit dem Offensichtlichen: Jedem einigermaßen am antiken griechischen Sport Interessierten ist bekannt, dass die bedeutendsten griechischen Agone - insbesondere die sogenannten „big four" der Periodos - in der Fachliteratur immer wieder „Heilige Spiele“ genannt werden. Doch auch schon in den antiken Quellen findet sich die Bezeichnung hieroi agones. ${ }^{2}$ Darüber hinaus besteht kein Zweifel, dass alleine aufgrund der Tatsache, dass Spiele, die, wie jene der Periodos, in Heiligtümern abgehalten wurden, in einem Naheverhältnis zu kultischen Abläufen standen, ja mit einer gewissen religiösen Aura versehen waren.

Aber bedeutet das auch, dass die Wettkämpfe selbst sakrale Handlungen darstellten, oder exakter formuliert, dass sie selbst bestimmte kultische Funktionen hatten? Dürfen wir sie wirklich zu Recht als „Gottesdienst“ bezeichnen. ${ }^{3}$

Zur religiösen, kultischen oder rituellen Bedeutung der sportlichen Agone gibt es mittlerweile eine unüberschaubare Menge an Literatur. ${ }^{4}$ Die Bandbreite reicht dabei

1. An dieser Stelle sei noch einmal Juan Ramón Carbó García und Jaime Alvar Ezquerra für die Einladung zur Tagung „Deporte, espectáculo y religión en la Antigüedad“, die hier herrschende anregende fachliche Atmosphäre und die großartige Gastfreundschaft mein herzlicher Dank ausgesprochen.

2. So etwa schon bei Pindar (Nem.2,4) oder später beispielsweise bei Diodor (14,109,3); auch bei Ulpian (Dig.3,2,4pr.) ist von certamina sacra die Rede, was als lateinische Variante verstanden werden kann.

3. So GEHRKE, H.-J.: "Olympia in der Geschichte", HEILMEUER, W.D. / KALTSAS, N. / GHERKE, H.-J. / HATZI, G.E. / BOCHER, S.(ed.): Mythos Olympia. Kult und Spiele, München/London/New York, 2012, 29-35 (32): „Zu dieser Religiosität der Griechen gehörte es auch, dass sich der Gottesdienst sehr gerne in Form von Wettbewerben, Agonen, vollzog."

4. Einen Überblick bieten etwa EVJEN, H.: "The Origins and Functions of Formal Athletic Competition in the Ancient world", COULSON, W. / KYRIELEIS, H. (ed.): Proceedings of an International Symposium on the Olympic Games, Athen, 1992, 95-104; GOLDEN, M.: Sport and Society in Ancient Greece, Cambridge, 1998, 10-23; oder PEMBERTON, E.: "Agones Hieroi. Greek Athletic Contests in their Religious Context", Nikephoros 13, 2000, 111-123, zu Olympia s. nach wie vor ULF, Chr. / WEILER, I.: "Der 
von Aussagen wie „alle Leibeserziehung war ursprünglich kultisch“ ${ }^{\text {“5 }}$ oder „sport is the ritual sacrifice of physical energy “ ${ }^{\prime}$ über Vorstellungen, dass durch sportliche Wettkämpfe Teilnehmer eines hieros gamos ausgewählt worden sein, ${ }^{7}$ und die Betonung der Rolle von Wettkämpfen im Totenkult ${ }^{8}$ bis zur Auffassung, dass die Wettkämpfe völlig profane Veranstaltungen waren. ${ }^{9}$ Es ist hier weder möglich noch meine Absicht, einen Überblick über all die bisher vorgestellten Ansätze und Meinungen zu geben. Auch werde ich mich nicht auf die ausufernde Frage des Ursprungs der Agone einlassen, die mit der nach der religiösen Bedeutung oft eng verbunden wird. Ich möchte hier ganz bewusst nicht auf Hypothesen zu quellenmäßig kaum fassbaren Zeiten eingehen und auch nicht versuchen, Überreste und Relikte eventueller kultischer Inhalte früherer oder gar ursprünglicher Wettkämpfe aufzuspüren und zu deuten.

Mein Zugang soll ein anderer sein. Es soll ganz konkret gefragt werden, ob in den antiken Quellen zu den Agonen, über die wir verfügen, tatsächliche kultische Funktionen der Wettkämpfe sichtbar werden oder nicht.

Vorweg möchte ich darauf hinweisen, dass grundsätzlich beide Möglichkeiten bekannt sind: Es gibt Wettkämpfe denen ein kultischer Charakter anzuhaften scheint und solche, wo sich dies nicht abzeichnet. So sind für die griechische Antike Wettkämpfe in physischen Leistungen überliefert, die zweifellos kultische oder zumindest rituelle Aspekte aufwiesen. ${ }^{10}$ Genannt seien hier allen voran Fackelläufe, in denen Feuer von einem Altar zu einem anderen transportiert wird. ${ }^{11}$ Hier kann jedoch etwa auch der Oschophorien Lauf am Fest der Skiren in Athen angeführt werden, bei dem Epheben mit einer Weinranke vom Tempel des Dionysos zum Tempel der Athena Skiras im Piräus um die Wette laufen, und über den uns Athenaios (Ath.11,495f-

Ursprung der antiken Olympischen Spiele in der Forschung. Versuch eines kritischen Kommentars", Stadion 6, 1980, 1-38; s. jüngst PATAY-HORVÁTH, A.: „Ein neuer Vorschlag zum Ursprung der Olympischen Spiele“, Gymnasium 123, 2016, 1-23.

5. DIEM, C.: Weltgeschichte des Sports und der Leibeserziehung, Stuttgart, 1960, 3.

6. SANSONE, D.: Greek Athletics and The Genesis of Sport, Berkeley/Los Angeles/London, 1988, 37, 65.

7. CORNFORD, F.: “The Origin of the Olympic Games," HARRISON, J. (ed.): Themis. A Study of the Social Origins of Greek Religion, Cambridge, 2010 (Erstpublikation 1927), 212-259.

8. MALTEN, L.: "Leichenspiel und Totenkult", $\operatorname{MDAI}(R)$ 38/39, 1923/24, 300-340.

9. So schon GARDINER, E.N.: "The alleged Kingship of the Olympic Victor", The Annual of the British School at Athens 22, 1916-18, 85-106; und ROSE, H.J.: “The Greek Agones”, Aberystwyth Studies 3, 1922, 1-24; später etwa PATRUCCO, R.: Lo sport nella Grecia antica, Florenz, 1972, 29.

10. Dazu etwa ROSE, H.J.: “The Greek Agones” (op. cit.), 13-14; RINGWOOD ARNOLD, I.C.: Agonistic Features of Local Greek Festivals Chiefly from Inscriptional Evidence, Diss. Columbia University, 1927, 12; SCANLON, T.F.: Eros and Greek Athletics, Oxford, 2002, 31.

11. Zu Fackelläufen s. AIGNER, Th. / MAURITSCH-BEIN, B. / PETERMANDL, W.: Laufen, Quellendokumentation zur Gymnastik und Agonistik im Altertum 7, Wien/Köln/Weimar, 2002, 419-422.

ARYs, 15, 2017 [33-48] ISSN 1575-166x 
496a) informiert. Als weiteres Beispiel darf hier wohl auch der Lauf der sytaphylodromoi (der sog. „Weinrebenlauf“) beim Kultfest der Karneia in Sparta genannt werden:

Anecd.Gr.Bekker, I p.305

Beim Karneenfest läuft jemand mit Kränzen umwunden und wünscht der Stadt Glück. Diesen verfolgen junge Männer, die sogenannten staphylodromoi. Wenn sie ihn einholen, erwarten sie nach Landessitte für die Stadt etwas Gutes, wenn sie ihn aber nicht einholen das Gegenteil.

(Üs. Therese Aigner)

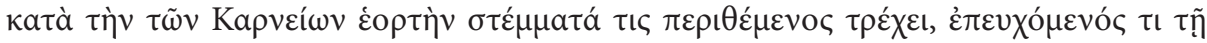

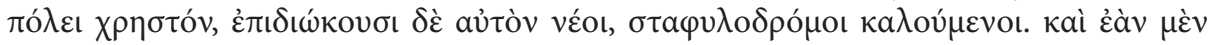

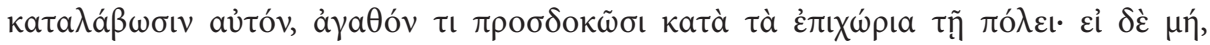
toủvavtíov.

Das gebotene Beispiel zeigt, dass es sich hier um rituelle Wettläufe ganz spezieller Art handelt, die nicht den Disziplinen entsprechen, wie sie bei den großen Agonen anzutreffen sind.

Auf der anderen Seite sind jedoch genauso gut Wettkämpfe nachzuweisen, bei denen kein kultischer Aspekt sichtbar wird. Ein berühmtes Beispiel wären die von den Phäaken für den noch unerkannten Odysseus veranstalteten Spiele (Hom. Od.8,100-255). Es ist auffällig, dass wir es hier nun sehr wohl mit Disziplinen zu tun haben, wie sie aus dem Sportprogramm der großen Agonen bekannt sind.

Zunächst sollen nun Quellenaussagen einer Betrachtung unterzogen werden, die man als Hinweise oder gar Belege für eine sakrale Bedeutung der Wettkämpfe bei den großen Agonen gewertet hat. Es soll überprüft werden, ob sich damit auch tatsächliche kultische Funktionen greifen lassen.

1. Agone - wie z.B. die „big four“ der Periodos, aber nicht nur diese - wurden in Heiligtümern und in sakralem Rahmen abgehalten. Diese Spiele waren damit in ein religiöses Umfeld eingebettet. Das ist in den Quellen wirklich zweifelsfrei belegt. Aber ist daraus schon eine kultische Funktion der Wettkämpfe ableitbar?

In Kulturen der verschiedensten Zeitstellungen rund um den Globus ist zu beobachten, dass Gruppen, die sich aus welchen Gründen auch immer versammeln, bei diesen Gelegenheiten häufig zur Austragung sportlicher Wettkämpfe neigen. In Samoa etwa veranstaltet die Bevölkerung Wettbewerbe, wenn sie zu traditionellen Festen - die auch religiöser Natur sein können - zusammenkommt. Auch in Ton- 
ga werden bei festlichen Anlässen traditionelle Wettbewerbe abgehalten. ${ }^{12}$ Für die Irokesen in Nordamerika werden besondere Bestattungszeremonien überliefert, zu denen man sich in regelmäßigen Abständen versammelte; und dabei kam es auch zur Veranstaltung von Wettkämpfen. ${ }^{13}$ Ähnliche Beobachtungen können auch beim sog. "messenger feast" der Eskimos Nordalaskas angestellt werden. ${ }^{14}$

Versammlungen können also allein schon ein hinreichender Grund für das Abhalten von Wettkämpfen sein. ${ }^{15}$ Und das gilt genauso für Versammlungen aus religiösen Gründen. Eine Beobachtung, die auch für die frühe griechische Welt formuliert wurde ${ }^{16}$ Das bedeutet aber, dass bei religiösen Zusammenkünften die Veranstaltung sportlicher Bewerbe denkbar ist, ohne dass diese Wettkämpfe selbst in irgendeiner Weise eine direkte Bindung zum religiösen Anlass aufweisen müssen. Eine kultische Funktion der Wettkämpfe kann daraus also keinesfalls abgeleitet werden.

2. Die Lage wäre anders zu beurteilen, wenn man nachweisen könnte, dass Darbietungen physischer Leistungen oder Wettkampfveranstaltungen den Anlass für die Entstehung der Heiligtümer dargestellt hätten. In der Folge könnte man dann eventuell - aber auch das ist natürlich nicht zwingend - ableiten, dass es ein ritueller oder kultischer Charakter dieser Wettkämpfe war, der dann zur Ausbildung der Heiligtümer führte. Es gibt eine Vielzahl hypothetischer Ansätze, die in diese Richtung gehen, doch können dafür keine wirklichen Beweise erbracht werden. ${ }^{17}$

Es ist nicht einmal möglich nachzuweisen, dass die Wettkämpfe am Anfang standen. Im Gegenteil, an Orten wie Olympia, Delphi und Isthmia scheint die archäologische Evidenz sogar dagegen zu sprechen. Spuren kultischer Aktivitäten lassen

12. SCHWARTZ, R.: "Autochthone Bewegungskultur in Polynesien: die Beispiele Western Samoa und Tonga”, Nikephoros 5, 1992, 183-215.

13. Ibidem.

14. WEILER, I.: "Joseph-François Lafitau (1681-1746) und die Anfänge einer komparativen Sportgeschichte", Nikephoros 6, 1993, 181-203.

15. ULF, Chr.: "Die Frage nach dem Ursprung des Sports, oder: weshalb und wie menschliches Verhalten anfängt, Sport zu sein”, Nikephoros 4, 1991, 13-30 (20-23), der dabei den Zweck des Sports ,als eine von vielen Möglichkeiten sozialer Kontaktaufnahme“ (18) betont und auf seine Fähigkeit, das Konfliktpotenzial zwischen Gruppen zu reduzieren, hinweist.

16. So auch DECKER, W.: Sport in der griechischen Antike. Vom minoischen Wettkampf bis zu den Olympischen Spielen, Hildesheim, 2012, 11: „Wo man sich versammelte, gehörte Sport im Allgemeinen dazu. Hier liegt ein Entstehungsanlass der großen Agone, ... " - Man kann auch heute leicht beobachten, dass, wenn etwa Gruppen von Jugendlichen zusammenkommen, es häufig schnell zu sportlichen Aktivitäten kommt.

17. SINN, U.: Olympia. Kult, Sport und Fest in der Antike, München, 1996, 20: „Wenn wir einmal alle Nachrichten über frühe Kultfeste zu Rate ziehen, finden wir dort zahlreiche Hinweise auf solches Wetteifern in gesellschaftlichem Rahmen." 
sich früher belegen, als jene von sportlichen Agonen. ${ }^{18}$ Es ist dabei natürlich zu berücksichtigen, dass Läufe, Ringkämpfe etc. nicht unbedingt archäologische Spuren hinterlassen müssen. Dennoch: An diesen Plätzen wird Kultisches vor Wettkämpfen greifbar. Keinesfalls lässt sich eine Entstehung der Heiligtümer aus Wettkämpfen beweisen und daraus auf eine kultische Funktion der Agone schließen.

3. Als nächsten Punkt soll kurz drauf eingegangen werden, dass schon in der Antike Spiele als hieroi agones bezeichnet werden konnten. Diese Benennung findet sich schon bei Pindar (Pind.Nem.2,4). Als technischer Begriff auf Siegerinschriften taucht diese Terminologie jedoch nicht vor 130 v.Chr. auf. ${ }^{19}$ Darin einen Hinweis auf kultische Funktionen der Agone zu sehen, ist nicht zulässig. Denn die Bezeichnung kann sich alleine aus der Tatsache ergeben, dass die Spiele im Umfeld von Heiligtümern bzw. religiösen Festen stattfanden.

4. Man hat auch versucht, im Homerischen Hymnos an Apoll ${ }^{20}(5,146-50)$ den kultischen Charakter von Agonen festzumachen. Darin ist zu lesen, die Ionier würden sich mit ihren Kindern und züchtigen Frauen in Delos versammeln. Weiter heißt es:

Freude bereiten sie dir [Apollon], denn sie denken an dich wenn der Wettstreit Anhebt mit Tänzen und Liedern und Faustkampf.

(Üs. Anton Weiher)

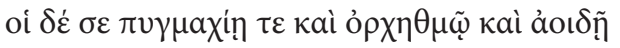

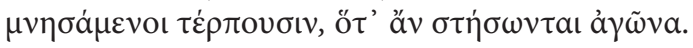

Wenn Scanlon meint, dies wäre der erste Verweis auf "an athletic festival performed specifically in honour of a god"21, kann ich ihm hier nicht völlig zustimmen. Ich würde eher von einem Fest zu Ehren eines Gottes sprechen, bei dem auch Agone abgehalten wurden. Ich sehe keine Bestätigung in dem Text, dass es der eigentliche Sinn des Festes in Delos war, den Gott mit Wettkämpfen zu erfreuen und zu ehren. Natürlich, freut sich der Gott über die Bewerbe. Aber er kann sich auch über Bewerbe

18. S. die in Anm.3 genannte Überblicksliteratur.

19. Vgl. Pemberton, E.: "Agones Hieroi (op. cit.), 112-116, "Olympia, Delphi, and Isthmia have yielded clear evidence for religious activity long before any athletic competitions were held" (112).

20. REMIJSEN, S.: "The So-Called 'Crown-Games': Terminology and Historical Context of the Ancient Catagories for Agones", ZPE 177, 2011, 97-107 (99).

21. Der Hymnos wird ins 8. aber auch ins 6. Jh.v.Chr. datiert. 
freuen, die nicht von kultischer Natur sind. Eine kultische Funktion der Wettkämpfe ist demnach auch weder zu erkennen noch zu erschließen.

5. In einem anderen antiken Text, bei Herdot $(2,91)$, findet man die Aussage, dass sich in der ägyptischen Stadt Chemmis ein Tempel befindet, in dem die Bewohner Perseus verehren.

Folgendes aber tun sie - auf griechische Art - für Perseus: Sie halten einen athletischen Wettkampf ab mit allen möglichen Kampfarten, wobei sie als Preise Vieh, Mäntel und Tierfelle aussetzen.

(Üs. Kai Brodersen)

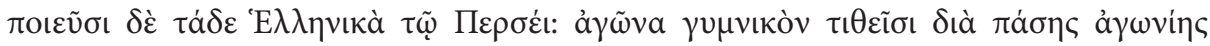

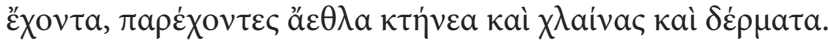

$$
[\ldots]
$$

Und weiter unten heißt es:

den athletischen Wettkampf hielten sie ab, da er (Perseus) dies befohlen habe.

(Üs. Kai Brodersen)

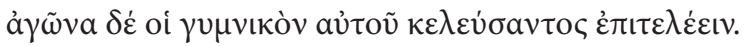

Wenn man nun in Rechnung stellt, dass für die Zeit des Herodot Wettkämpfe durchaus schon als eine übliche Erscheinung in Heiligtümern gelten können, ist die Ansicht, dass sich Perseus selbst so etwas auch für sein Fest gewünscht habe, nicht sehr überraschend. Allerdings kann sich Perseus durchaus auch profane Wettkämpfe gewünscht haben. Nur weil der Gott Wettkämpfe bei seinem Fest haben möchte, bedeutet das noch nicht, dass diese Wettkämpfe genuin kultisch sind bzw. kultische Funktion haben müssen.

6. Eine Stelle, die immer wieder herangezogen wird, um die kultische Rolle der Wettkämpfe bei den Olympischen Spiele zu bestätigen, findet sich in Philostrats peri gymnastikes 5 .

Auf den Stadionlauf ist man so verfallen: Als die Eleer, wie ihr Gesetz vorschreibt, opferten, lagen zwar auf dem Altar die Opfergaben bereit, Feuer jedoch war an sie noch nicht angelegt. Ein Stadion aber waren die Läufer vom Altar entfernt, und davor stand ein Priester als Schiedsrichter mit einer Fackel. Und der Sieger zündete das Opfer an und ging als Olympionike hervor.

(Üs. Julius Jüthner) 


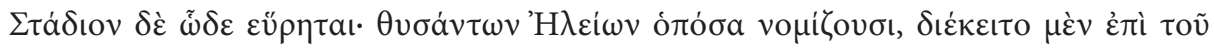

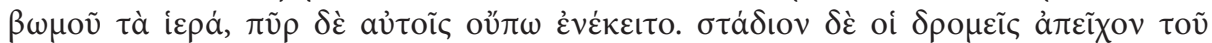

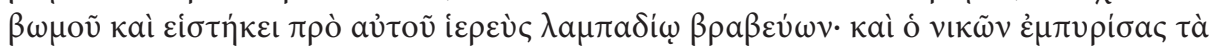

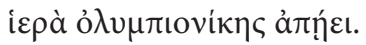

Es steht völlig außer Zweifel, dass sich diese Passage nicht auf die Zeit Philostrats und etwaige eigene Beobachtungen der Vorgänge in Olympia bezieht, sondern die Ansicht des Verfassers vom Ursprung der Spiele in einer viele Jahrhunderte zurückliegenden Vergangenheit wiedergibt. Damit fällt die Stelle als Nachweis eines tatsächlichen Sachverhaltes aus. Um den Realitätsgehalt der Stelle einzuschätzen, kann man den Text auch ein wenig weiterlesen und sich ansehen, was Philostrat zur Einführung des diaulos zu berichten weiß.22 Kurz, es ist nicht möglich mit dieser Philostrat-Stelle tatsächliche kultische Funktionen der Wettkämpfe in Olympia zu belegen.

7. Die räumliche Lage der früheren Stadien in den Heiligtümern von Olympia, Isthmia und Nemea ist ein weiterer Gesichtspunkt, der in der gegenständlichen Frage angesprochen werden kann. Denn alle drei liegen näher am Zentrum des jeweiligen Heiligtums als ihre jüngeren Nachfolgebauten. Sie scheinen sogar in gewisser Weise auf die Zentren der Heiligtümer ausgerichtet zu sein. Allerdings gibt es keine Nachweise, dass die Stadien exakt bei einem Altar oder dergleichen endeten.

Dieser archäologische Befund reicht nicht aus, um einen kultisch aufgeladenen Lauf, wie ihn etwa Philostrat schildert, zu beweisen. Dass in der Folge die jüngeren Stadien ab dem 5. Jahrhundert dann weiter weg von den Zentren der Heiligtümer angelegt werden, zeigt jedenfalls eher, dass zu dieser späteren Zeit keine direkte kultische Anbindung an diese bestand, als dass damit eine solche für die früheren Stadien bestätigt wird.

8. Es ist für unsere Fragestellung beachtenswert, dass in den Spielen der Periodos als Preise lediglich Kränze verliehen wurden. Es ist nicht zu leugnen, dass Kränze auch in religiösen Zeremonien eine große Rolle spielten. ${ }^{23}$ Außerdem gibt es die antike Überlieferung (Paus.5,15,3; Schol.vet.Pind Ol.3,60), dass die Olivenzweige für den olympischen Siegeskranz von einem Baum im Heiligtum abgeschnitten wurden; und

22. SCANLON, T.F.: Eros and Greek Athletics (op.cit.), 27.

23. Philostr.gym.6: „Nachdem aber die Eleer geopfert hatten, mussten auch die Festgesandten der Hellenen opfern, die sich einfanden. Damit aber deren Ankunft nicht ohne Zeremoniell vor sich gehe, liefen die Läufer vom Altare weg ein Stadion weit, wie um die Hellenen einzuladen, und kehrten wiederum ebendahin zurück, als meldeten sie, dass Hellas also mit Freuden kommen wolle. Soviel nun über den Ursprung des Doppellaufs [diaulos]“. 
zwar von einem pais amphitales, d.h. einem Knaben, dessen beide Eltern noch am Leben sein mussten. Das klingt nun schon sehr nach religiösem Tun und man hat darin Überreste einer ursprünglich kultischen Rolle der Wettkämpfe gesehen. ${ }^{24}$ Auf die Frage der ursprünglichen Inhalte der Wettkämpfe möchte ich aber hier, wie gesagt, nicht eingehen. Für meinen Zugang ist jedoch wichtig, dass für die ganze Zeit, in der der Brauch, Kränze als Preis zu vergeben, bezeugt ist, keine damit verbundenen konkreten kultischen Aspekte nachweisbar sind. Von der anderen Seite betrachtet, scheint es dagegen nahezu zu erwarten zu sein, dass auch bei profanen Spielen, die in einem Heiligtum abgehalten wurden, die Preise von diesem Heiligtum beigesteuert wurden. Auf dieser Grundlage kann daher für die Wettkämpe keine kultische Funktion festgemacht werden.

9. Eine weitere auffallende Besonderheit der griechischen Agone ist es, dass für die grundsätzlich freigeborenen Athleten bei Regelübertretungen die Prügelstrafe vorgesehen war. Eine Strafe, die normalerweise auf Kinder und Sklaven beschränkt blieb. Ich kann hier keine Erklärung für diesen auffälligen Sachverhalt anbieten. Eine Zusammenstellung und genaue Auswertung sämtlicher verfügbarer Quellen hinsichtlich dieser Frage, kann hier vermutlich noch weitere Erkenntnisse bringen. Jedenfalls genügt die Prügelstrafe meines Erachtens nicht, eine kultische Funktion der Wettkämpfe nachzuweisen.

10. Eine andere besondere Art der Bestrafung, über die Pausanias (Paus.5,21) informiert, ist die Aufstellung der sog. Zanes (Zeusstatuen) in Olympia, die aus Strafgeldern korrupter Athleten zu errichten waren. Ich würde zurückhaltend sein, hier von einer "sacred sanction“ ${ }^{\text {“25 }} \mathrm{zu}$ sprechen; vor allem dann, wenn damit impliziert wird, diese Praxis sei begründet in einem sakralen Charakter der Wettkämpfe. Dass in wichtigen oder prestigeträchtigen Situationen spektakuläre Sanktionen gegen Fehlverhalten gesetzt werden, scheint naheliegend. So wird etwa auch in der Athenaion Politeia (Aristot.Athen.Pol.55,5) erwähnt, dass Thesmotheten, als Strafe für Geschenkannahme, eine goldene Statue weihen müssen. Hieraus wird man wohl nicht ableiten, dass die Thesmotheten ein kultisches Amt innehatten. In der Tatsache, dass eine Zeusstatue aus Strafgeldern zu bezahlen ist, vermag ich dementsprechend auch keinen Hinweis zu sehen, dass die Wettkämpfe kultische Funktionen erfüllten.

24. S. dazu BLECH, M.: Studien zum Kranz bei den Griechen, Berlin/New York, 1982, 302-312.

25. So etwa VALAVANIS, P.: "Thoughts on the Historical Origins of the Olympic Games and the Cult of Pelops in Olympia", Nikephoros 19, 2006, 137-152 (145). 
11. Zwei weitere Aspekte sollen hier gemeinsam besprochen werden: die sog. athletische Nacktheit und die Altersklassen bei den Wettkämpfen. Beide sind gut belegt. Und beide werden des Öfteren als Relikte einer ursprünglichen Funktion der Wettkämpfe als Initiationsriten gesehen. ${ }^{26}$ Man kann diese Überlegungen von ursprünglich rituellen Praktiken wahrscheinlich finden oder nicht, ${ }^{27}$ was ich an dieser Stelle aufzeigen möchte ist, dass die Agone, ab der Zeit, in der sie in den Quellen greifbar werden, jedenfalls keinerlei Funktionen dieser Art erkennen lassen. Dafür fehlt, soweit ich sehe, sowohl im Falle der athletischen Nacktheit als auch der Altersklassen jeglicher Hinweis.

12. Auf den sog. Olympischen Eid möchte ich hier ebenfalls kurz eingehen. Auch seine Existenz kann jedoch eine kultische Bedeutung der Wettkämpfe nicht bezeugen. Bei Pausanias (Paus.5,24,9-10) wird der Eid beschrieben, den die Athleten in Olympia schwören müssen und in dem sie sich verpflichten, nicht gegen die Regeln verstoßen zu werden. Die Frage ist nun: Bedeutet das Schwören eines heiligen Eides, bestimmte Bestimmungen einzuhalten, gleichzeitig, dass diese Bestimmungen selbst sakraler Natur sein müssen? Sicher nicht.

13. Auch der Ausschluss von Frauen vom olympischen Agon wurde mit einer kultischen Bedeutung der Spiele in Zusammenhang gebracht. ${ }^{28}$ Doch muss das bei Pausanias erwähnte Verbot der Anwesenheit verheirateter Frauen (Paus.5,6,7) nicht unbedingt allein mit den Wettkämpfen zu tun haben. Vielmehr scheint es wahrscheinlich, ein derartiges Verbot mit Kulthandlungen für Zeus zu verbinden. ${ }^{29}$ Für derartige Ausschlüsse von Frauen von Heiligtümern oder bestimmten Kultveranstaltungen können Vergleichsbeispiele beigebracht werden. ${ }^{30}$ Außerdem, sollte dieses Verbot tatsächlich ausschließlich mit den Wettkämpfen zu begründen sein - was sich keinesfalls beweisen lässt -, wäre damit noch nicht gesagt, dass es ihre kultische Funktion war, die ein Anwesendsein der Frauen unmöglich machte.

26. SCANLON, T.F.: Eros and Greek Athletics (op.cit.), 38.

27. Zur Nacktheit s. BONFANTE, L.: "Nudity as Costume in Greek Art", AJA 93, 1989, 543-579; vgl. z.B. auch KYLE, D.G.: Sport and Spectacle in the ancient world, Oxford, 2007, 118-120; zu Altersklassen s. schon JEANMAIRE, H.: Couroi et Courètes, Lille, 1939 (ND New York 1975), 413-415; BRELICH, A.: Gli eroi greci, Rom, 1958, 210-213; vgl. etwa auch ULF, Chr. / WEILER, I.: “Der Ursprung (op. cit.), 21.

28. Zu meiner Meinung in diesen Punkten s. PETERMANDL, W.: "Überlegungen zur Funktion der Altersklassen bei den griechischen Agonen", Nikephoros 10, 1997, 135-147; PETERMANDL, W.: "The Introduction of Athletic Nudity - Fact or Fiction?” Nikephoros 27 (im Druck).

29. So z.B. VALAVANIS, P.: “Thoughts (op. cit.), 145-147; der jedoch nur davon spricht, dass dies „originally (Kursivsetzung: WP) had a cult significance."

30. Paus.5,6,7 spricht von einem Verbot für Frauen zum Agon zu kommen „oder auch nur an den für sie verbotenen Tagen“ den Alpheios zu überschreiten. 
14. Das gleiche Argument, das gerade herangezogen wurde, um den Ausschluss der Frauen als Nachweis von kultischen Funktionen der Agone zurückzuweisen, kann auch hinsichtlich der sog. Ekecheirie - des Olympischen Festfriedens - geltend gemacht werden. Wie beim Frauenausschluss müsste wohl auch hinsichtlich der Ekecheirie zunächst einmal bewiesen werden, dass sie direkt mit den Wettkämpfen zu verknüpfen ist. Letztlich erscheint es mir viel plausibler, zunächst einmal an eine Schutzeinrichtung für das Kultfest und seine Teilnehmer zu denken. ${ }^{31}$ Damit kann auch die Ekecheirie nicht als Beweis eines besonderen sakralen Charakters der Agone herangezogen werden.

Nach diesem Überblick über eine Reihe von im Quellenmaterial enthaltenen Anknüpfungspunkten, die sich als Stütze für die Ansicht von einer kultischen Funktion der Agone als haltlos erweisen, sollen in der Folge nun kurz einige Beobachtungen vorgestellt werden, die deutlich gegen einen kultischen Charakter der Wettkämpfe sprechen.

1. Das stärkste Argument scheint mir zu sein, dass in der doch relativ reichen Überlieferung zu den großen, bedeutenden Spielen, soweit ich sehe, keine Hinweise auf irgendwelche eindeutig kultischen Funktionen der Wettkämpfe selbst greifbar werden.

2. Das Gleiche gilt für das mit der Abwicklung der Wettkämpfe befasste Personal. So sind wir zwar über die olympischen Hellanodiken relativ gut unterrichtet, doch sind für sie keine kultischen oder auch nur als religiös zu bezeichnenden Aufgaben bezeugt. ${ }^{32}$

Auf der anderen Seite ist jedoch auch nicht bekannt, dass das für die religiösen Belange zuständige Personal irgendwie in die Wettkämpfe eingebunden gewesen wäre. ${ }^{33}$

3. Schon vor fast 100 Jahren hat man weiters zu Recht darauf aufmerksam gemacht, ${ }^{34}$ dass die ausgeprägte Entwicklung des Wettkampfprogramms mit fortschreitender Zeit nicht zu einem bei kultischen Praktiken zu beobachtenden Konservativismus passt.

31. So etwa Herakles Heiligtümer, vgl. dazu etwa MOURATIDIS, J: "Heracles at Olympia and the exclusion of women from the ancient Olympic Games", Journal of Sport History 11, 1984, 41-55.

32. So wie etwa auch ein besonderer Festfrieden für die Mysterien in Eleusis belegt ist; s. etwa MYLONAS, G.E.: Eleusis and the Eleusinian Mysteries, Princeton, 1961, 244. auch SCHWEIGERT, E., „Greek Inscriptions", Hesperia 8, 1939, 1-49 (10).

33. Eine Ausnahme stellt vielleicht IvO 14 dar (den Hinweis auf die Inschrift verdanke ich Paul Christesen), doch vermag ich der Bronzeplatte aufgrund ihres fragmentarischen Zustandes wenig Konkretes zu entnehmen.

34. So etwa auch JÜTHNER, J. / BREIN, F.: Die athletischen Leibesübungen der Griechen. I. Geschichte der Leibesübungen, Sitzungsberichte der österreichischen Akademie der Wissenschaften, phil.-hist.Kl. 249,1, Graz/Wien/Köln, 1965, 75; SIEWERT, P. “The Olympic Rules”, COULSON, W. / KYRIELEIS, H. (ed.): Proceedings of an International Symposium on the Olympic Games. 5-9 September 1988, Athens, 1992, 113-11 (116); GOLDEN, M.: Sport and Society (op. cit.), 15. 
4. Zum Schluss möchte ich noch ein weiteres Argument anfügen, das mir bislang nicht wirklich berücksichtigt worden zu sein scheint. Es liegt in der bekannten Kritik der relativ frühen Autoren, Xenophanes und Euripides. ${ }^{35}$ In beiden Texten, werden die Agone in einer Weise kritisiert, die keinesfalls zu Veranstaltungen passt, die starke kultische oder religiöse Aspekte besitzen bzw. gar Aufgaben erfüllen. Wobei es hier, meines Erachtens, keine Rolle spielt, wie man die jeweiligen Sprecher bzw. deren Einstellung zum Sport einordnen möchte ${ }^{36}$.

Xenophanes (6./5. Jh.) - Xenoph.frg.2 DK (= Ath.10,413f-414c)

Aber wenn einer durch die Schnelligkeit seiner Füße den Sieg erränge oder als einer, der das Pentathlon bestreitet, dort wo das Heiligtum des Zeus liegt neben dem Strom Pisa in Olympia, oder als Ringer oder als Meister des schmerzreichen Faustkampfs oder des furchtbaren Wettbewerbs, den sie Pankration nennen, wäre er zwar für die Bürger glanzvoller anzuschauen und würde auch den deutlich erkennbaren Ehrenplatz bei den Agonen erhalten und wohl auch Brot aus dem Gemeinbesitz und ein Geschenk von der Stadt, das ihm ein Kleinod wäre, selbst wenn er mit seinen Pferden siegte, würde er alles das erhalten - und doch wäre er dessen nicht so würdig wie ich. Denn besser als die Kraft von Männern oder Pferden ist meine Geisteskraft. Das wird vielmehr ganz willkürlich praktiziert, und es ist auch nicht gerecht, Körperkraft der guten Geisteskraft vorzuziehen. Denn weder wenn ein guter Boxer unter dem Volk ist, noch einer, der gut im Pentathlon oder im Ringen ist, noch einer, der sich auszeichnet durch die Schnelligkeit der Füße - was ja gerade von allen Wettbewerben das am meisten geachtete ist unter allen Kraftstücken der Männer - wird deshalb die Stadt in einem höheren Grad von Eunomia leben. Nur wenig Grund zur Freude hat die Stadt darüber, wenn einer siegt, der an den Ufern des Flusses Pisa antritt, denn das füllt die Vorratskammern der Stadt nicht.

(Üs. nach Joachim Latacz)

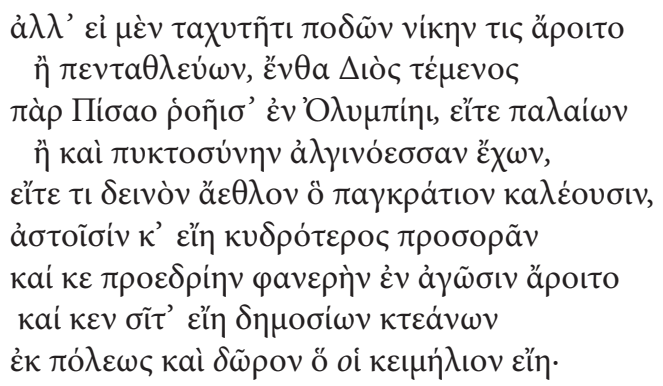

35. ROSE, H.J.: “The Greek Agones” (op. cit.), 17; RINGWOOD ARNOLD, I.C.: Agonistic Features (op. cit.), 12; später s. etwa JÜTHNER, J. /BREIN, F.: Die athletischen Leibesübungen (op. cit.), 75.

36. Wobei allerdings einzuräumen ist, dass beide Texte erst als Fragmente bei Athenaios (2./3. Jh.n.Chr.) überliefert sind. 


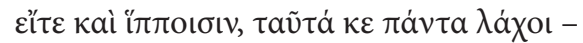

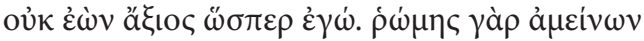

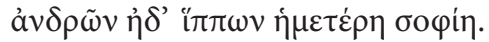

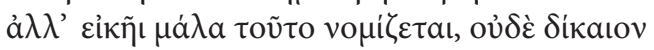

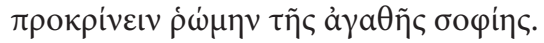

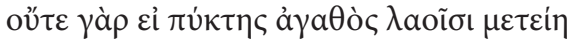

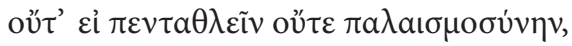

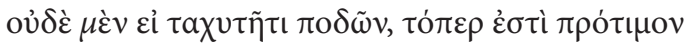

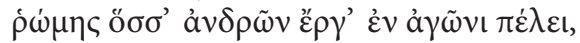

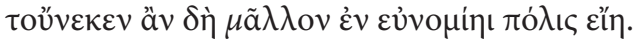

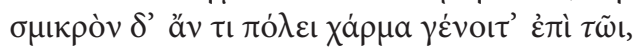

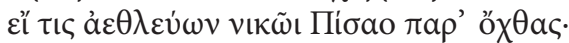

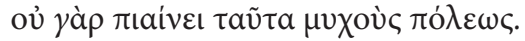

Würde man die Sieger einer kultisch aufgeladenen Veranstaltung so kritisieren? Hätten die religiösen Aspekte einfach übergangen werden können? Die gleichen Fragen können ebenso an die Stelle bei Euripides herangetragen werden:

Euripides (5.Jh.) - Eur. Frg.282 (351) (= Ath.10,413c-f)

Ja, von den tausend Übeln, die in Hellas nisten, ist doch das allerschlimmste jenes der Athleten.

...

Denn welcher Meisterringer, Läufer, Diskoswerfer, wer auch, der ganz genau des Gegners Kinn getroffen, hat durch den Siegeskranz dem Vaterland genützt? Behauptet man mit Disken sich im Handgemenge vorm Feinde - oder jagt man nicht vielmehr den Feind im Kampf mit schweren Waffen aus der Heimatflur? Kein andrer stellt so dumm sich an im Schwertgefecht Mann gegen Mann! Die Klugen und die Tüchtigen soll man bekränzen, alle, die den Staat vortrefflich durch Scharfsinn und Gerechtigkeit zu lenken wissen, auch durch Verhandeln ihrem Staat den Krieg ersparen, nach außen wie nach innen: Damit leisten sie Vorzügliches für ihre Stadt und alle Griechen! (Üs. Dietrich Ebener)

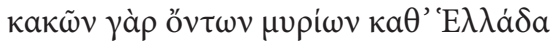

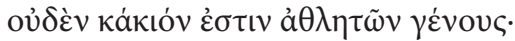
...

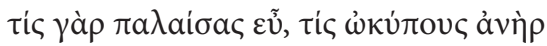

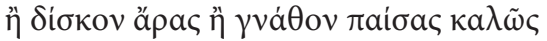




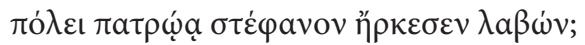

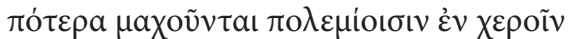

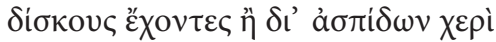

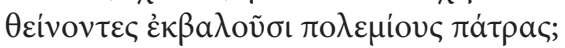

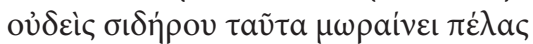

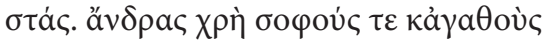

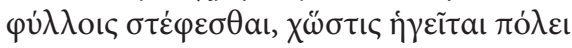

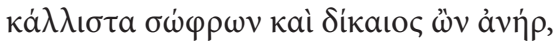

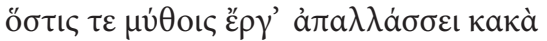

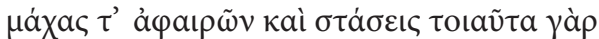

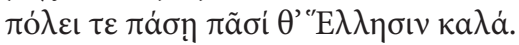

Zum Schluss kommend muss ich zwar eingestehen, dass der hier vorgenommene Überblick durchaus als etwas gewagt oder gar kühn zu bezeichnen ist. Musste er doch selbstverständlich weit davon entfernt bleiben, ein ausgesprochen facettenreiches und vielbehandeltes Themenfeld auch nur in Ansätzen umfassend zu skizzieren sowie im Detail auf in der Fachliteratur formulierte Meinungen und Thesen einzugehen. Es sollte aber versucht werden, ein paar grundlegende Aspekte herauszustellen. Wichtig war es mir dabei, Überlegungen zum Ursprung der Agone, die in den meisten Abhandlungen den Blick schnell auf einen sehr hypothetischen Boden führen, bewusst auszuklammern. Auf diese Weise ist ein deutlicheres Bild von den Aussagen der Quellen zu gewinnen, die sich auf die jeweils aktuellen Spiele beziehen.

Als Resultat wäre zusammenzufassen, dass sich konkrete kultische Funktionen der Wettkämpfe, soweit ich sehe, zurzeit nicht nachwiesen lassen, und dass - im Gegenteil - die Auswertung des verfügbaren Quellenmaterials sogar gegen die Annahme kultischer Funktionen der Wettkämpfe spricht.

\section{LITERATUR}

Aigner, Th./Mauritsch-Bein, B./Petermande, W.: Laufen, Quellendokumentation zur Gymnastik und Agonistik im Altertum 7, Wien/Köln/Weimar, 2002.

Blech, M.: Studien zum Kranz bei den Griechen, Berlin/New York, 1982.

Bonfante, L.: "Nudity as Costume in Greek Art", AJA 93, 1989, 543-579.

Brelich, A.: Gli eroi greci, Rom, 1958.

Cornford, F.: "The Origin of the Olympic Games," Harrison, J. (ed.): Themis. A Study of the Social Origins of Greek Religion, Cambridge, 2010 (Erstpublikation 1927), 212-259.

DeCKeR, W.: Sport in der griechischen Antike. Vom minoischen Wettkampf bis zu den Olympischen Spielen, Hildesheim, 2012.

Diem, C.: Weltgeschichte des Sports und der Leibeserziehung, Stuttgart, 1960.

Evjen, H.: "The Origins and Functions of Formal Athletic Competition in the Ancient world", Coulson, W./Kyrieleis, H. (ed.): Proceedings of an International Symposium on the Olympic Games, Athen, 1992, 95-104. 
Gardiner, E.N.: “The alleged Kingship of the Olympic Victor", The Annual of the British School at Athens 22, 1916-18, 85-106.

Gehrke, H.-J.: "Olympia in der Geschichte”, Heilmeuer, W.D./Kaltsas, N./Gherke, H.-J./ Hatzi, G.E./Bocher, S.(ed.): Mythos Olympia. Kult und Spiele, München/London/New York, 2012, 29-35.

Golden, M.: Sport and Society in Ancient Greece, Cambridge, 1998.

Jeanmaire, H.: Couroi et Courètes, Lille, 1939 (ND New York 1975).

Jüthner, J./BreIn, F.: Die athletischen Leibesübungen der Griechen. I. Geschichte der Leibesübungen, Sitzungsberichte der österreichischen Akademie der Wissenschaften, phil.hist.Kl. 249,1, Graz/Wien/Köln, 1965.

KYLE, D.G.: Sport and Spectacle in the ancient world, Oxford, 2007.

Malten, L.: "Leichenspiel und Totenkult", $M D A I(R)$ 38/39, 1923/24, 300-340.

Mouratidis, J: "Heracles at Olympia and the exclusion of women from the ancient Olympic Games", Journal of Sport History 11, 1984, 41-55.

MylonAs, G.E.: Eleusis and the Eleusinian Mysteries, Princeton, 1961.

PAtay-Horváth, A.: „Ein neuer Vorschlag zum Ursprung der Olympischen Spiele“, Gymnasium 123, 2016, 1-23.

PAtrucco, R.: Lo sport nella Grecia antica, Florenz, 1972.

Pemberton, E.: "Agones Hieroi. Greek Athletic Contests in their Religious Context", Nikephoros 13, 2000, 111-123.

Petermand, W.: "Überlegungen zur Funktion der Altersklassen bei den griechischen Agonen", Nikephoros 10, 1997, 135-147.

PetermandL, W.: “The Introduction of Athletic Nudity - Fact or Fiction?” Nikephoros 27 (im Druck).

Remijsen, S.: “The So-Called 'Crown-Games': Terminology and Historical Context of the Ancient Catagories for Agones", ZPE 177, 2011, 97-107.

Ringwood Arnold, I.C.: Agonistic Features of Local Greek Festivals Chiefly from Inscriptional Evidence, Diss. Columbia University, 1927.

Rose, H.J.: “The Greek Agones”, Aberystwyth Studies 3, 1922, 1-24.

SAnsone, D.: Greek Athletics and The Genesis of Sport, Berkeley/Los Angeles/London, 1988

SCANLON, T.F.: Eros and Greek Athletics, Oxford, 2002.

Schwartz, R.: "Autochthone Bewegungskultur in Polynesien: die Beispiele Western Samoa und Tonga", Nikephoros 5, 1992, 183-215.

SChWEIGERT, E., “Greek Inscriptions”, Hesperia 8, 1939, 1-49.

Siewert, P. “The Olympic Rules", Coulson, W./Kyrieleis, H. (ed.): Proceedings of an International Symposium on the Olympic Games. 5-9 September 1988, Athen, 1992, 113-117

Sinn, U.: Olympia. Kult, Sport und Fest in der Antike, München, 1996.

Ulf, Chr.: "Die Frage nach dem Ursprung des Sports, oder: weshalb und wie menschliches Verhalten anfängt, Sport zu sein", Nikephoros 4, 1991, 13-30.

Ulf, Chr./Weiler, I.: "Der Ursprung der antiken Olympischen Spiele in der Forschung. Versuch eines kritischen Kommentars", Stadion 6, 1980, 1-38.

Valavanis, P.: "Thoughts on the Historical Origins of the Olympic Games and the Cult of Pelops in Olympia”, Nikephoros 19, 2006, 137-152.

WeILER, I.: "Joseph-François Lafitau (1681-1746) und die Anfänge einer komparativen Sportgeschichte", Nikephoros 6, 1993, 181-203. 